\title{
ChMusic: A Traditional Chinese Music Dataset for Evaluation of Instrument Recognition
}

\author{
Xia Gong \\ School of Music, Shandong University of Technology, Zibo, China, sdlggx@ @dut.edu.cn \\ Yuxiang Zhu \\ No.2 High School (Baoshan) of East China Normal University, Shanghai, China, 3447535189@qq.com \\ Haidi Zhu \\ Shanghai Institute of Microsystem and Information Technology, Chinese Academy of Sciences, Shanghai, China, hdzhu@mail.sim.ac.cn \\ Haoran Wei \\ Department of Electrical and Computer Engineering, University of Texas at Dallas Richardson, USA, haoran.wei@utdallas.edu
}

\begin{abstract}
Musical instruments recognition is a widely used application for music information retrieval. As most of previous musical instruments recognition dataset focus on western musical instruments, it is difficult for researcher to study and evaluate the area of traditional Chinese musical instrument recognition. This paper collected a traditional Chinese music dataset for training model and performance evaluation, named ChMusic. This dataset is free and publicly available, 11 traditional Chinese musical instruments and 55 traditional Chinese music excerpts are recorded in this dataset. Then an evaluation standard is proposed based on ChMusic dataset. With this standard, researchers can compare their results following the same rule, and results from different researchers will become comparable. Additional Keywords and Phrases: Chinese instruments, music dataset, Chinese music, machine learning, evaluation of musical instrument recognition
\end{abstract}

\section{INTRODUCTION}

Musical instruments recognition is an important and fascinating application for music information retrieval(MIR). Deep learning is great tool for image processing $[1,2,3]$, video processing $[4,5,6]$, natural language processing $[7,8,9]$, speech processing $[10,11,12]$ and music processing $[13,12,15]$ applications and has demonstrated better performance compared with previous approaches. While deep learning based approaches rely on proper datasets to train model and evaluate performance.

Though there are already several musical instruments recognition dataset, most of these datasets only covers western musical instruments. For examples, Good-sounds dataset [16] has 12 musical instruments consisting of flute, cello, clarinet, trumpet, violin, sax alto, sax tenor, sax baritone, sax soprano, oboe, piccolo and bass. Another musical instruments recognition dataset called [17] has 20 musical instruments consisting of accordion, banjo, bass, cello, clarinet, cymbals, drums, flute, guitar, mallet percu ssion, mandolin, organ, piano, saxophone, synthesizer, trombone, trumpet, ukulele, violin, and voice. Very few attention has been made to other culture's musical instruments recognition. Even for the very limited researches base on Chinese musical instruments recognition [18,19], dataset used for these research are not publicly available. Without an open access Chinese musical instruments dataset, Another critical problem is that researchers cannot evaluate their model performance by a same standard, so results reported from their papers are not comparable.

To deal with the problems mentioned above, a traditional Chinese music dataset, named ChMusic, is proposed to help training Chinese musical instruments recognition models and then conducting performance evaluation. So the major contributions of this paper can be concluded as:

1) Propose a traditional Chinese music dataset for Chinese traditional instrument recognition, named ChMusic.

2) Come up with an evaluation standard to conduct Chinese traditional instrument recognition on ChMusic dataset.

3) Propose baseline approach for Chinese traditional instrument recognition on ChMusic dataset, code for this baseline is https://github.com/HaoranWeiUTD/ChMusic

The rest of the paper is organized as follows: Section 2 covers details of ChMusic dataset. Then, the corresponding evaluation standard to conduct Chinese traditional instrument recognition on ChMusic dataset are described in Section 3. Baseline for Musical Instrument Recognition on ChMusic Dataset is proposed in Section 4. Finally, the paper is concluded in Section 5.

\section{CHMUSIC DATASET}

ChMusic is a traditional Chinese music dataset for training model and performance evaluation of musical instrument recognition. This dataset cover 11 musical instruments, consisting of Erhu, Pipa, Sanxian, Dizi, Suona, Zhuiqin, Zhongruan, Liuqin, Guzheng, Yangqin and Sheng, the indicating numbers and images are shown on Figure 1 respectively.

Each musical instrument has 5 traditional Chinese music excerpts, so there are 55 traditional Chinese music excerpts in this dataset. The name of each music excerpt and the corresponding musical instrument is shown on Table 1 Each music excerpt only played by one musical instrument in this dataset. 


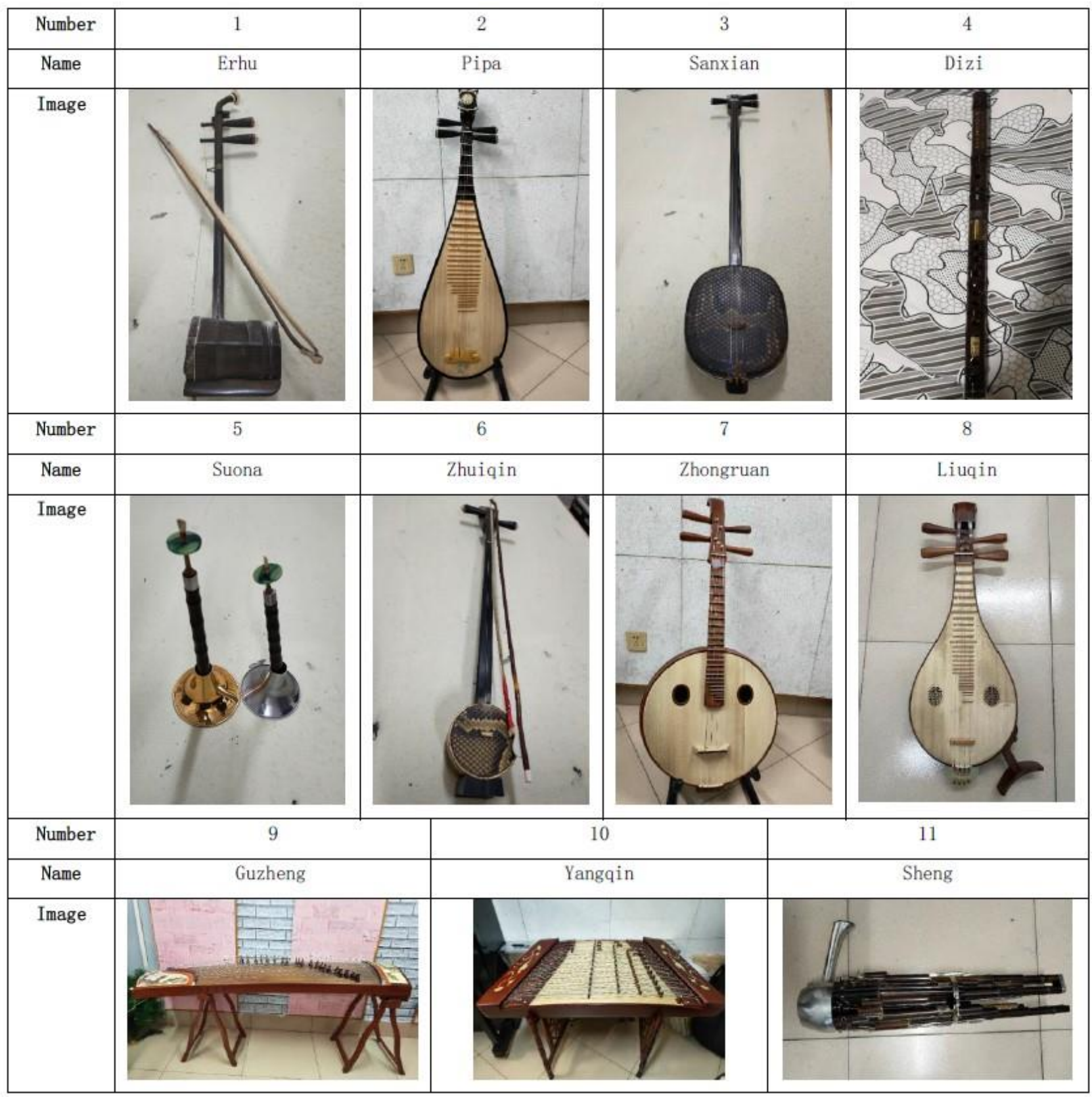

Figure 1. Example of a traditional Chinese instrument.

Each music excerpt is saved as a .wav file. The name of these files follows the format of "x.y.wav", "x" indicates the instrument number, ranging from 1 to 11 , and "y" indicates the music number for each instrument, ranging from 1 to 5 . These files are recorded by dual channel, and has sampling rate of $44100 \mathrm{~Hz}$. The duration of these music excerpts are between 25 to 280 seconds.

This ChMusic dataset can be download from Baidu Wangpan by link: pan.baidu.com/s/13e-6GnVJmC3tcwJtxed3-g with passwords: xk23, or from google drive: drive.google.com/file/d/1rfbXpkYEUGw5h_CZJtC7eayYemeFMzij/view?usp=sharing

\section{EVALUATION OF MUSICAL INSTRUMENT RECOGNITION ON CHMUSIC DATASET}

The diagram of musical instrument recognition can be simplified as Figure 2. Figure 2 consisting of two stages, corresponding to training stage and testing stage respectively. Training data is used for training stage to train a musical instrument recognition model. Then this model is tested by testing data during testing stage. 
Table 1: Musics Played By Traditional Chinese Instruments

\begin{tabular}{|c|c|c|}
\hline $\begin{array}{c}\text { Instrument } \\
\text { Name }\end{array}$ & $\begin{array}{c}\text { Music } \\
\text { Number }\end{array}$ & Music Name \\
\hline \multirow[t]{5}{*}{ Erhu } & 1 & "Ao Bao Xiang Hui" \\
\hline & 2 & "Mu Yang Gu Niang" \\
\hline & 3 & "Xiao He Tang Shui" \\
\hline & 4 & "Er Xing Qian Li" \\
\hline & 5 & "Yu Jia Gu Niang", \\
\hline \multirow[t]{5}{*}{ Pipa } & 1 & "Shi Mian Mai Fu" excerpts \\
\hline & 2 & "Su" excerpts \\
\hline & 3 & "Zhu Fu" excerpts \\
\hline & 4 & "Xian Zi Yun" excerpts \\
\hline & 5 & "Ying Hua" \\
\hline \multirow[t]{5}{*}{ Sanxian } & 1 & "Kang Ding Qing Ge" \\
\hline & 2 & "Ao Bao Xiang Hui" \\
\hline & 3 & "Gan Niu Shan" \\
\hline & 4 & "Mao Zhu Xi De Hua Er Ji Xin Shang" \\
\hline & 5 & "Nan Ni Wan" \\
\hline \multirow[t]{5}{*}{ Dizi } & 1 & "Hong Mei Zan" \\
\hline & 2 & "Shan Hu Song" \\
\hline & 3 & "Wei Shan Hu" \\
\hline & 4 & "Xiu Hong Qi" \\
\hline & 5 & "Xiao Bai Yang" \\
\hline \multirow[t]{5}{*}{ Suona } & 1 & "Hao Han Ge" \\
\hline & 2 & "She Yuan Dou Shi Xiang Yang Hua" \\
\hline & 3 & "Yi Zhi Hua" \\
\hline & 4 & "Huang Tu Qing" excerpts \\
\hline & 5 & "Liang Zhu" excerpts \\
\hline \multirow[t]{5}{*}{ Zhuiqin } & 1 & "Jie Nian" excerpt 1 \\
\hline & 2 & "Jie Nian" excerpt 2 \\
\hline & 3 & "Hong Sao" excerpt \\
\hline & 4 & "Jie Nian" excerpt 3 \\
\hline & 5 & "Zi Mei Yi Jia" excerpt \\
\hline \multirow[t]{5}{*}{ Zhongruan } & 1 & "Yun Nan Hui Yi" excerpt 1 \\
\hline & 2 & "Yun Nan Hui Yi" excerpt 2 \\
\hline & 3 & "Yun Nan Hui Yi" excerpt 3 \\
\hline & 4 & "Yun Nan Hui Yi" excerpt 4 \\
\hline & 5 & "Yun Nan Hui Yi" excerpt 5 \\
\hline \multirow[t]{5}{*}{ Liuqin } & 1 & "Chun Dao Yi He" excerpt 1 \\
\hline & 2 & "Yu Ge" excerpt \\
\hline & 3 & "Chun Dao Yi He" excerpt 2 \\
\hline & 4 & "Yu Hou Ting Yuan" excerpt \\
\hline & 5 & "Jian Qi" excerpt \\
\hline \multirow[t]{5}{*}{ Guzheng } & 1 & "Lin Chong Ye Ben" excerpt 1 \\
\hline & 2 & "Zhan Tai Feng" excerpt \\
\hline & 3 & "Yu Zhou Chang Wan" \\
\hline & 4 & "Lin Chong Ye Ben" excerpt 2 \\
\hline & 5 & "Han Jiang Yun" \\
\hline \multirow[t]{5}{*}{ Yangqin } & 1 & "Zuo Zhu Fa Lian Xi Qu" \\
\hline & 2 & "Fen Jie He Xian Lian Xi Qu" \\
\hline & 3 & "Dan Yin Yu He Yin Jiao Ti Lian Xi Qu" \\
\hline & 4 & "Lun Yin Lian Xi Qu" \\
\hline & 5 & "Da Qi Luo Gu Qing Feng Shou" \\
\hline \multirow[t]{5}{*}{ Sheng } & 1 & "Gua Hong Deng" excerpt \\
\hline & 2 & "Qin Wang Po Zhen Yue" excerpt \\
\hline & 3 & "Shui Ku Yin Lai Jin Feng Huang" \\
\hline & 4 & "Shan Xiang Xi Kai Feng Shou Lian" \\
\hline & 5 & "Xi Ban Ya Dou Niu Wu Qu" \\
\hline
\end{tabular}




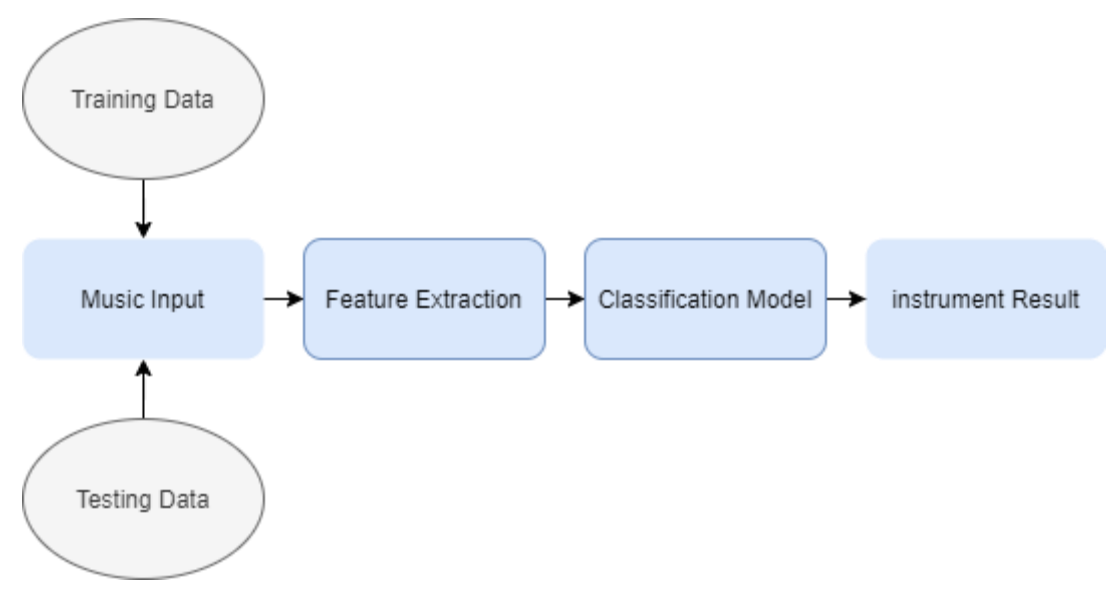

Figure 2. Diagram of musical instrument recognition.

The widely used features for musical instrument recognition include linear predictive cepstral coefficient (LPCC) [20] and melscale frequency cepstral coefficients (MFCC) [21]. The commonly used classification models include k-nearest neighbors (KNN) model [18], Gaussian mixture model(GMM)[22] , support vector machine (SVM) model[23], hidden markov model (HMM) [24] and deep learning based models [25] .

In ChMusic Dataset, music with number 1 to 4 of each instrument are used as training dataset, music with number 5 of each instrument are used as testing dataset. Every music is cut into 5 seconds clips without overlap, the ending part of each music which is shorter than 5 seconds is discarded. Each 5 seconds clips need to make a prediction for musical instrument classification result.

$$
\text { Accuracy }=\frac{\text { CorrectClassifiedClips }}{\text { AllClipsFromTestingData }}
$$

Classification accuracy will be the evaluation metrics for musical instrument recognition on ChMusic Dataset. And confusion matrix is also recommended to present the result.

\section{BASELINE FOR MUSICAL INSTRUMENT RECOGNITION ON CHMUSIC DATASET}

Baseline for musical instrument recognition on ChMusic dataset is described in this section. Machine learning [26, 27, 28, 29, $30,31]$ and deep learning $[32,33,34,35,36,37,38,39,40]$ are useful tools to deal with classification and recognition related problems $[41,42,43,44,45,46,47,48,49,50]$, baseline approach is conducted by machine learning approach. Twenty dimensions MFCCs are extracted from each frame, and KNN is used for frame level data training and testing. As each test clip have 5 minutes in duration, a majority voting is conducted to get final classification result from frame-level results within the 5 minutes test clip.

KNN models with various $\mathrm{K}$ value are compared. Accuracy for KNN models with each $\mathrm{K}$ value are presented in Table 2 then confusion matrix for $\mathrm{K}=3, \mathrm{~K}=5, \mathrm{~K}=9$ and $\mathrm{K}=15$ are presented in Figure 3, Figure 4, Figure 5 and Figure 6 respectively.

Table 2: Accuracy for Knn Models with Various K Value

\begin{tabular}{|c|c|}
\hline Model & Accuracy(\%) \\
\hline KNN (K=3) & 93.57 \\
\hline KNN (K=5) & 93.57 \\
\hline KNN (K=9) & 93.57 \\
\hline KNN (K=15) & 94.15 \\
\hline
\end{tabular}




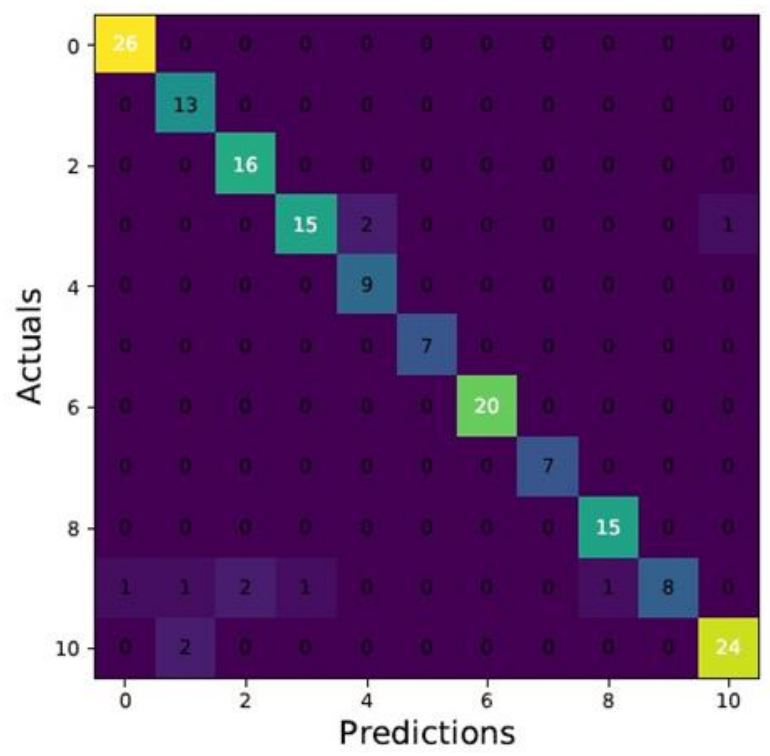

Figure 3. Confusion matrix of $\mathrm{KNN}$ model with $\mathrm{K}=3$.

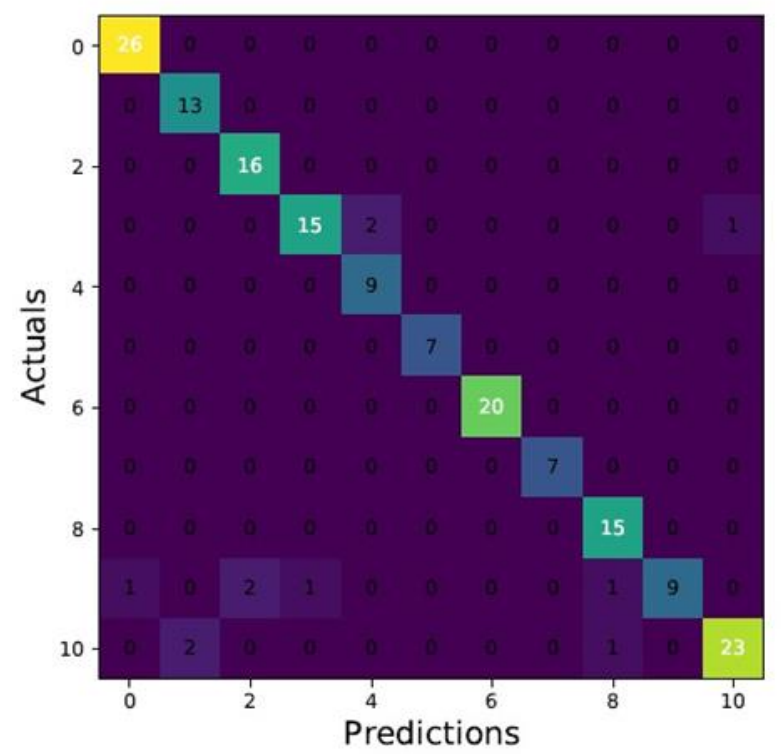

Figure 4. Confusion matrix of KNN model with $\mathrm{K}=5$. 


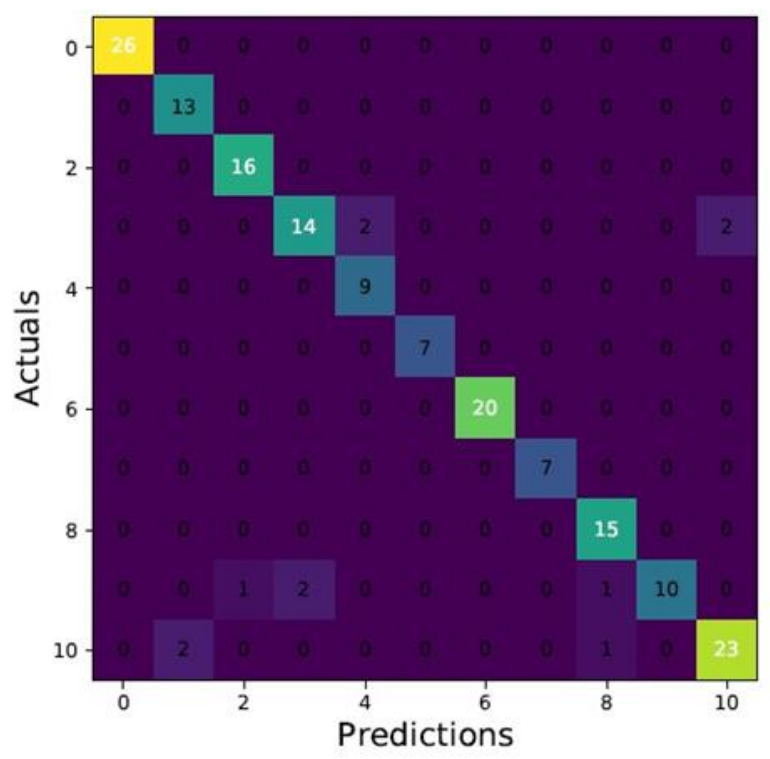

Figure 5. Confusion matrix of KNN model with $\mathrm{K}=9$.

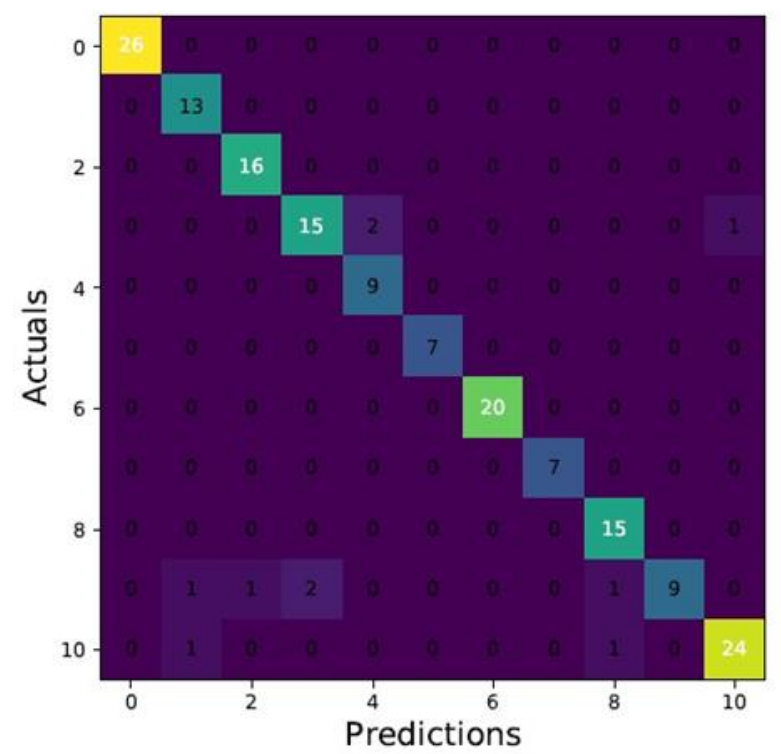

Figure 6. Confusion matrix of KNN model with $\mathrm{K}=15$.

\section{CONCLUSION}

Musical instruments recognition is an interesting application for music information retrieval. While most of previous musical instruments recognition dataset focus on western musical instruments, very few works have been conducted on the area of traditional Chinese musical instrument recognition. This paper proposes a traditional Chinese music dataset for training model and performance evaluation, named ChMusic. With this dataset, researchers can download it for free. Eleven traditional Chinese musical instruments and 55 traditional Chinese music excerpts are covered in this dataset. Then an evaluation standard is proposed based on ChMusic dataset. With this standard, researchers can compare their results following the same rule, and results from different researchers will become comparable. 


\section{ACKNOWLEDGMENT}

Thanks for Zhongqin Yu, Ziyi Wei, Baojin Qin, Guangyu Zhao, Qiuxiang Wang, Xiaoqian Zhang, Jiali Yao, Zheng Gao, Ke Yan, Menghao Cui and Yichuan Zhang for playing musics and helping to collect this ChMusic dataset.

\section{REFERENCES}

[1] A. Azarang and H. Ghassemian, "Application of fractional-order differentiation in multispectral image fusion," Remote sensing letters, vol. 9, no. 1, pp. 91-100, 2018.

[2] A. Azarang and N. Kehtarnavaz, "A generative model method for unsupervised multispectral image fusion in remote sensing," Signal, Image and Video Processing, pp. 1-9, 2021.

[3] H. Wei, A. Sehgal, and N. Kehtarnavaz, "A deep learning-based smartphone app for real-time detection of retinal abnormalities in fundus images," in RealTime Image Processing and Deep Learning 2019, vol. 10996. International Society for Optics and Photonics, 2019, p. 1099602.

[4] H. Zhu, H. Wei, B. Li, X. Yuan, and N. Kehtarnavaz, "A review of video object detection: Datasets, metrics and methods," Applied Sciences, vol. 10, no. 21, p. 7834, 2020.

[5] H. Wei and N. Kehtarnavaz, "Simultaneous utilization of inertial and video sensing for action detection and recognition in continuous action streams," IEEE Sensors Journal, vol. 20, no. 11, pp. 6055-6063, 2020.

[6] H. Zhu, H. Wei, B. Li, X. Yuan, and N. Kehtarnavaz, "Real-time moving object detection in high-resolution video sensing," Sensors, vol. 20, no. 12, p. $3591,2020$.

[7] X. Sun, L. Jiang, M. Zhang, C. Wang, and Y. Chen, "Unsupervised learning for product ontology from textual reviews on ecommerce sites," in Proceedings of the 2019 2nd International Conference on Algorithms, Computing and Artificial Intelligence, 2019, pp. 260-264.

[8] L. Jiang, O. Biran, M. Tiwari, Z. Weng, and Y. Benajiba, "End-to-end product taxonomy extension from text reviews," in 2019 IEEE 13th International Conference on Semantic Computing (ICSC). IEEE, 2019, pp. 195-198.

[9] X. Li, L. Vilnis, D. Zhang, M. Boratko, and A. McCallum, "Smoothing the geometry of probabilistic box embeddings," in International Conference on Learning Representations, 2018.

[10] R. Su, F. Tao, X. Liu, H. Wei, X. Mei, Z. Duan, L. Yuan, J. Liu, and Y. Xie, “Themes informed audio-visual correspondence learning," arXiv preprint arXiv:2009.06573, 2020.

[11] Y. Zhang, Y. Long, X. Shen, H. Wei, M. Yang, H. Ye, and H. Mao, "Articulatory movement features for short duration text-dependent speaker verification," International Journal of Speech Technology, vol. 20, no. 4, pp. 753-759, 2017.

[12] N. Alamdari and N. Kehtarnavaz, "A real-time smartphone app for unsupervised noise classification in realistic audio environments," in 2019 IEEE International Conference on Consumer Electronics (ICCE). IEEE, 2019, pp. 1-5.

[13] E. Benetos, S. Dixon, Z. Duan, and S. Ewert, “Automatic music transcription: An overview,” IEEE Signal Processing Magazine, vol. 36, no. 1, pp. 20-30, 2018.

[14] R. Lu, K. Wu, Z. Duan, and C. Zhang, "Deep ranking: Triplet matchnet for music metric learning," in 2017 IEEE International Conference on Acoustics, Speech and Signal Processing (ICASSP). IEEE, 2017, pp. 121-125.

[15] P. Shreevathsa, M. Harshith, A. Rao et al., "Music instrument recognition using machine learning algorithms," in 2020 International Conference on Computation, Automation and Knowledge Management (ICCAKM). IEEE, 2020, pp. 161-166.

[16] G. Bandiera, O. Romani Picas, H. Tokuda, W. Hariya, K. Oishi, and X. Serra, "Good-sounds. org: A framework to explore goodness in instrumental sounds," in Proceedings of the 17th International Society for Music Information Retrieval Conference; 2016 Aug 7-11; New York City (NY).[Canada]: ISMIR; 2016. p. 4149. International Society for Music Information Retrieval (ISMIR), 2016.

[17] E. Humphrey, S. Durand, and B. McFee, “Openmic2018: An open data-set for multiple instrument recognition.” in ISMIR, 2018 , pp. 438-444.

[18] J. Shen, "Audio feature extraction and classification of the chinese national musical instrument," Computer Digital Engineering, 2012.

[19] H. Shi, W. Xie, T. Xu, X. Zhu, and Q. Li, "esearch and ealization of identification methods on ethnic musical instruments," Shanxi Dianzi Jishu, 2020.

[20] J. Picone, "Fundamentals of speech recognition: A short course," Institute for Signal and Information Processing, Mississippi State University, 1996.

[21] H. Wei, Y. Long, and H. Mao, "Improvements on selfadaptive voice activity detector for telephone data," International Journal of Speech Technology, vol. 19, no. 3, pp. 623-630, 2016.

[22] Y. Anzai, Pattern recognition and machine learning. Elsevier, 2012.

[23] Y. Long, W. Guo, and L. Dai, “An sipca-wccn method for svm-based speaker verification system," in 2008 International Conference on Audio, Language and Image Processing. IEEE, 2008, pp. 1295-1299.

[24] P. Herrera-Boyer, A. Klapuri, and M. Davy, "Automatic classification of pitched musical instrument sounds," in Signal processing methods for music transcription. Springer, 2006, pp. 163-200.

[25] H. Wei and N. Kehtarnavaz, "Determining number of speakers from single microphone speech signals by multi-label convolutional neural network," in IECON 2018-44th Annual Conference of the IEEE Industrial Electronics Society. IEEE, 2018, pp. 2706-2710.

[26] C. Li, L. Tian. "Association between resting-state coactivation in the parieto-frontal network and intelligence during late childhood and adolescence," American Journal of Neuroradiology. 2014, 35(6), pp:1150-1156.

[27] Z. Zhang, C. Sun, C. Li, M. Sun. "Vibration based bridge scour evaluation: A data-driven method using support vector machines," Structural monitoring and maintenance. 2019, 6(2), pp:125-145. 
[28] M. Sun, C. Li, H. Zha. "Inferring private demographics of new users in recommender systems," In Proceedings of the 20th ACM International Conference on Modelling, Analysis and Simulation of Wireless and Mobile Systems, 2017, pp. 237-244.

[29] X. Li, L. Meng, Y. Tan, J. Zhang, W. Wan, H. Zhang. "Deep semantic segmentation-based multiple description coding," Multimedia Tools and Applications. 2021, 80(7), pp:10323-10337.

[30] H. Shao, J. Li, J. Zhang, H. Yu, J. Sun. “Eye-based Recognition for User Identification on Mobile Devices,” ACM Transactions on Multimedia Computing, Communications, and Applications (TOMM). 2020, 16(4), pp:1-9.

[31] H. Li, L. Meng, J. Zhang, Y. Tan, Y. Ren, H. Zhang. "Multiple description coding based on convolutional auto-encoder," IEEE Access. 2019, 7, pp:2601326021.

[32] Y. Long, R. He. “The SHNU System for the CHiME-5 Challenge," Proc. CHiME 2018 Workshop on Speech Processing in Everyday Environments. 2018, pp: 64-66.

[33] K. Killamsetty, C. Li, C. Zhao, R. Iyer, F. Chen. "A Reweighted Meta Learning Framework for Robust Few Shot Learning," arXiv preprint arXiv:2011.06782, 2020.

[34] H. Wei, F. Tao, R. Su, S. Yang, J. Liu. "Ensemble Chinese End-to-End Spoken Language Understanding for Abnormal Event Detection from audio stream," arXiv preprint arXiv:2010.09235. 2020.

[35] L. Peng, A. Jiang, H. Wei, B. Liu, M. Wang. “Ensemble single image deraining network via progressive structural boosting constraints, ” Signal Processing: Image Communication. 2021, p.116460.

[36] Y. Shi, J. Zhou, Y. Long, Y. Li, H. Mao. “Addressing Text-Dependent Speaker Verification Using Singing Speech,” Applied Sciences, 2019,9 (13), 2636.

[37] Y. Long, Y. Li, B. Zhang. "Offline to online speaker adaptation for real-time deep neural network based LVCSR systems," Multimedia Tools and Applications,2018, 77(21), pp:28101-28119.

[38] C. Zhao, C. Li, J. Li, F. Chen. "Fair meta-learning for few-shot classification,” In 2020 IEEE International Conference on Knowledge Graph (ICKG), 2020, pp. 275-282.

[39] H. Wei and N. Kehtarnavaz, "Semi-supervised faster renn-based person detection and load classification for far field video surveillance," Machine Learning and Knowledge Extraction, vol. 1, no. 3, pp. 756-767, 2019.

[40] J. Chen, J. Kong, H. Sun, H. Xu, X. Liu, Y. Lu, and C. Zheng, "Spatiotemporal interaction residual networks with pseudo3d for video action recognition," Sensors, vol. 20, no. 11, p. 3126, 2020.

[41] H. Wei, M. Laszewski, and N. Kehtarnavaz, "Deep learning based person detection and classification for far field video surveillance," in 2018 IEEE 13 th Dallas Circuits and Systems Conference (DCAS), 2018, pp. 1-4.

[42] Y. Long, S. Wei, Q. Zhang, C. Yang. "Large-Scale Semi-Supervised Training in Deep Learning Acoustic Model for ASR,” IEEE Access, 2019, (7), pp:133615-133627.

[43] Y. Liu, K. Wang, G. Li, and L. Lin, "Semantics-aware adaptive knowledge distillation for sensor-to-vision action recognition," IEEE Transactions on Image Processing, 2021.

[44] J.-K. Tsai, C.-C. Hsu, W.-Y. Wang, and S.-K. Huang, "Deep learning-based real-time multiple-person action recognition system,” Sensors, vol. 20, no. 17, p. $4758,2020$.

[45] Z. Ahmad and N. Khan, "Cnn based multistage gated average fusion (mgaf) for human action recognition using depth and inertial sensors, ” IEEE Sensors Journal, 2020.

[46] C. Chen, R. Jafari, and N. Kehtarnavaz, "Utd-mhad: A multimodal dataset for human action recognition utilizing a depth camera and a wearable inertial sensor," in 2015 IEEE International conference on image processing (ICIP). IEEE, 2015, pp. 168-172.

[47] H. Wei, R. Jafari, and N. Kehtarnavaz, "Fusion of video and inertial sensing for deep learning-based human action recognition," Sensors, vol. 19, no. 17, p. 3680, 2019.

[48] C. Chen, R. Jafari, and N. Kehtarnavaz, "Improving human action recognition using fusion of depth camera and inertial sensors," IEEE Transactions on HumanMachine Systems, vol. 45, no. 1, pp. 51-61, 2014.

[49] N. Dawar and N. Kehtarnavaz, "Continuous detection and recognition of actions of interest among actions of non-interest using a depth camera," in 2017 IEEE International Conference on Image Processing (ICIP). IEEE, 2017, pp. 4227-4231.

[50] Z. Liu, L. Meng, Y. Tan, J. Zhang, H. Zhang. "Image compression based on octave convolution and semantic segmentation," Knowledge-Based Systems, 2021, p.107254. 